\title{
Physical Basis of Lognormals in Reliability for Microelectronics With Deposited Metal Layers
}

This paper was downloaded from TechRxiv (https://www.techrxiv.org).

\section{LICENSE}

CC BY 4.0

SUBMISSION DATE / POSTED DATE

03-03-2022 / 03-03-2022

\section{CITATION}

eng, genghmun (2022): Physical Basis of Lognormals in Reliability for Microelectronics With Deposited Metal Layers. TechRxiv. Preprint. https://doi.org/10.36227/techrxiv.19295165.v1

DOI

10.36227/techrxiv.19295165.v1 


\title{
Physical Basis of Lognormals in Reliability for Microelectronics With Deposited Metal Layers
}

\author{
Genghmun Eng \\ FFRDC Physical Sciences Laboratories, Retired Senior Scientist
}

February 14, 2022

\begin{abstract}
Historically, many electronic device lifetimes were empirically modeled with Lognormals and Weibulls, but a physical basis was elusive until now. We show Lognormals naturally arise for long thin conducting metal layers deposited on substrates. Key elements are: (1) an applied voltage $V_{M}$ across the metal layer ends; (2) good thermal connectivity through the substrate to a constant temperature $T_{B}$ baseplate; and (3) a metal layer failure mechanism that has an $a c$ tivation energy $U_{M}$. Since these (1)-(3) conditions are common to many microelectronic devices, Lognormals should also be applicable to a wide variety of similar situations.

Ohm's Law, $V_{M}=I R_{M}$, with power dissipation $P=I^{2} R_{M}$, produces extra heat, giving a metal layer temperature $T_{L}>T_{B}$, which sets a baseline $\mathcal{F}\left(T_{L}\right)$ failure rate. What we derive here is that having a Gaussian variation in metal layer geometry produces Lognormal distributions for the metal layer lifetimes, which provides a new physical basis for using Lognormals in microelectronic device reliability.

Email: geng001@socal.rr.com

PACS: 85.40.Qx, 72.15.Eb, 73.40.Ns
\end{abstract}

\section{Introduction}

Probability Density Functions (pdfs) for electronic device lifetimes have been modeled using Lognormals, Exponentials (random failures), Gaussians (wearout), and Weibulls, but a physical basis for a using Lognormals or Weibulls has remained elusive until now. Here, we show why long thin conducting metal layers, deposited as wires on a non-conducting bulk substrate, should exhibit Lognormal pdfs.

This derivation relies on these metal layers having: (1) An Arrhenius-type failure mechanism with activation energy $U_{M} ;(2)$ An applied voltage $V_{M}$ across the metal layer, and (3) Good thermal connectivity through the substrate down to a underlying heat-sink held at temperature of $T_{B}$.

Many modern-day devices and components obey the above (1)-(3) conditions, and Lognormal pdfs have often been empirically observed, such as in $L E D$ Numeric Display Failures ${ }^{\mathbf{1 - 2}}$ and in semiconductor device accelerated aging $\mathrm{data}^{\mathbf{3 - 5}}$. However, this Lognormal $p d f$ connection was apparently not well known as late as $1957^{6}$, as that was prior to transistor miniaturization technology.

When failure rates $\mathcal{F}$ obey an Arrhenius plot, that process is characterized by activation energy $U_{M}$, giving a nearly constant negative slope on a $\ln (\mathcal{F})$ vs $\beta \equiv\left(1 / k_{B} T\right)$ plot, with $k_{B}$ being Boltzmann's Constant. This signature indicates a diffusion-driven failure mechanism due to unwanted atomic migration. A nominal wire temperature $T_{L}$ sets a baseline $\mathcal{F}\left(T_{L}\right)$ metal layer lifetime. We then show how a Gaussian variation in metal layer geometry results in a Lognormal distribution for metal layer lifetimes, providing a Lognormal physical basis. 


\section{Lognormals for Metal Layers}

When deposited metal layers atop a substrate are used for wire interconnects, their failure rate $\mathcal{F}$ is often set by an activation energy $U_{M}$, and a nominal operating temperature $T_{L}$ :

$$
\mathcal{F}_{M}\left(T_{L}\right)=\mathcal{F}_{\infty} \exp \left[-U_{M} / k_{B} T_{L}\right],
$$

where $\mathcal{F}_{\infty}$ is an asymptotic material constant. If an anomalous wire section has a different local temperature $T \neq T_{L}$, its failure rate will be:

$$
\begin{aligned}
& \mathcal{F}(T)=\mathcal{F}_{M} \exp \left[-U_{M}\left(\frac{1}{k_{B} T}-\frac{1}{k_{B} T_{L}}\right)\right], \\
& \ln \left[\mathcal{F}(T) / \mathcal{F}_{M}\right]=-U_{M}\left(\frac{1}{k_{B} T}-\frac{1}{k_{B} T_{L}}\right) .
\end{aligned}
$$

Our key point is any device geometry change that alters the local temperature from $T_{L}$ to $T$, also changes the wire lifetime, making $\mathcal{F}(T)$ a function of geometry. If those geometry changes are due to manufacturing variations, as characterized by a Gaussian random variable $X$ with zero mean and a fixed standard deviation, once it is shown that:

$$
\ln \left[\mathcal{F}(T) / \mathcal{F}_{M}\right]=+\mathbf{K} X\left[1+\vartheta\left(X^{2}\right)\right]
$$

for small $X$ and some constant $\mathbf{K}$, then it immediately follows that $\mathcal{F}(T)$ is a Lognormal distribution.

\subsection{Geometrical and Thermal Factors}

Figure 1 shows two different deposited metal layers atop a non-conducting substrate, as used in wire interconnects between devices. In (1a), the normal metal layer section has length $L_{a}$, depth $d_{o}$, a normal width $w_{o}$, and a cross-sectional area of $A_{o}=w_{o} d_{o}$. In (1b), the anomalous metal layer section has the same $\left\{L_{a} ; d_{o}\right\}$ length and depth, but with improper width $w_{a}$, and a different cross-sectional area of $A_{a}=$ $w_{a} d_{o}$. Each $L_{a}$ section is also part of a longer thin deposited wire of total length:

$$
L_{T O T}=\left(2 L_{n}+L_{a}\right) \text {, }
$$

with the other $L_{n}$ lengths having a normal metal layer geometry.

Figure 2 shows a side view, with a metal layer of thickness $d_{o}$ at $Z=0$, sitting atop a substrate of thickness $Z_{B}$, which is underlain by a heat-sink at $Z=Z_{B}$ held at temperature $T_{B}$. When the metal layer is at temperature $T \neq T_{B}$, a linear temperature profile forms across the substrate between $Z=0$ and $Z=Z_{B}$.

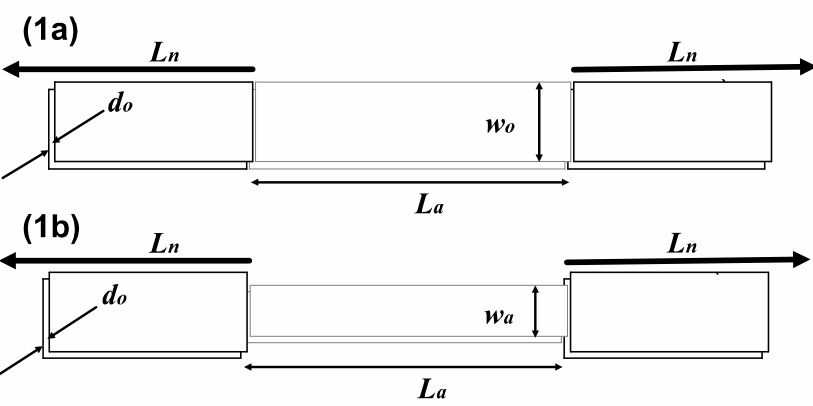
Figure 1: Nominal (1a) vs Anomalous (1b) Metal Layer Geometries. The anomalous portion is assumed to have an improper width $\boldsymbol{w a}$, spanning a length $\boldsymbol{L} \boldsymbol{a}$, as compared to a normal width $\boldsymbol{w o}$. To the left and right of this length $\boldsymbol{L} \boldsymbol{a}$ are two long normal $\boldsymbol{w}_{\boldsymbol{o}}$ sections, each of length $\boldsymbol{L}_{n}$, giving a total length of (2 $\left.\boldsymbol{L}_{n}+\boldsymbol{L a}\right)$.

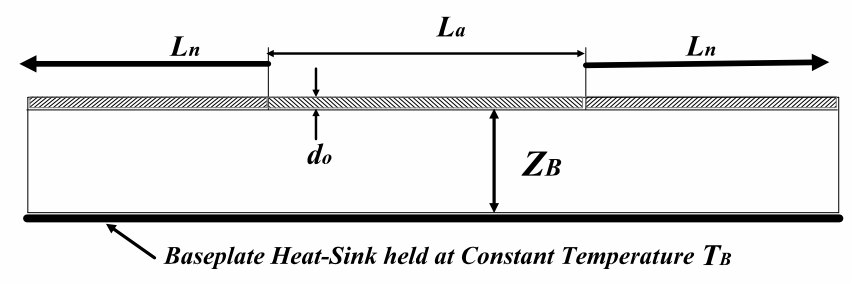

Figure 2: Side View of Deposited Metal Layer atop a Substrate. Central portion of length $\boldsymbol{L} a$, may have a normal $(\boldsymbol{w o})$ or anomalous (wa) width. It abuts two long normal $\boldsymbol{w}_{\boldsymbol{o}}$ sections of length $\boldsymbol{L} \boldsymbol{n}$, for a total length $(2 \boldsymbol{L} \boldsymbol{n}+\boldsymbol{L} \boldsymbol{a})$. The Metal Layer depth is $\boldsymbol{d} \boldsymbol{o}$, and it sits atop a substrate of thickness $Z_{\boldsymbol{B}}$, with a heat-sink at the bottom that is held at a constant temperature $\boldsymbol{T}_{\boldsymbol{B}}$. 
The Wiedemann-Franz Law applies ${ }^{\mathbf{7}}$ to the metal layer electrical resistance:

$$
\begin{aligned}
& {\left[K_{M} /\left(\sigma_{M} T\right)\right] \equiv \Lambda_{o}=\frac{\pi^{2}}{3}\left(\frac{k_{B}}{e}\right)^{2}} \\
& \quad \approx 2.445 \times 10^{-8}(\text { Watt }- \text { ohms }) / K^{2},
\end{aligned}
$$

due to electrons near the metal Fermi Level contributing both to the metal layer $K_{M}$ thermal conductivity $[W$ atts $/($ meter $-K)]$ and its $\sigma_{M}$ electrical conductivity $[1 /($ ohm - meter $)]$, where $\Lambda_{o}$ is the Lorentz Number. For metals, $K_{M}$ is fairly temperature independent, making $\sigma_{M}=\sigma_{M}(T)$, with $T$ in Kelvin.

When the metal layer carries a current $I$, its power dissipation $P[W a t t s]$ needs to be conducted out of the metal layer, through the substrate, down to the $Z=Z_{B}$ heat-sink. The heat flux $J_{S}\left[\right.$ Watts $\left./ \mathrm{m}^{2}\right]$ out of the metal layer, through the substrate, is controlled by the $K_{S}$ substrate thermal conductivity $[$ Watts / $($ meter $-K)]$.

For the anomalous wire in (1b), its $L_{n}$ normal geometry sections are assumed to have a boundary condition of $T(Z=0)=T_{n}$ and $T\left(Z=Z_{B}\right)=T_{B}$, giving an in-substrate temperature profile of:

$T(Z)=\left(T_{n}\right)-\left(T_{n}-T_{B}\right) Z / Z_{B}$,

with a temperature gradient and $J_{n}^{S}$ heat flux of:

$$
\nabla T(Z)=-\left(T_{n}-T_{B}\right) / Z_{B},
$$

$J_{n}^{S}=-\frac{K_{S}}{Z_{B}}\left(T_{n}-T_{B}\right)$. (1b) will have:

$$
\begin{aligned}
& T(Z)=\left(T_{a}\right)-\left(T_{a}-T_{B}\right) Z / Z_{B}, \\
& J_{a}^{S}=-\frac{K_{S}}{Z_{B}}\left(T_{a}-T_{B}\right) .
\end{aligned}
$$

Under constant $V_{M}$ conditions, the presence of an anomalous wire section changes the equilibrium temperatures for both the normal $w_{o}$ and the anomalous $w_{a}$ width sections, compared to the totally uniform wire case of $(\mathbf{1 a})$.

\subsection{Electrical Factors}

Applying a voltage $V_{M}$ across a metal layer with resistance $R_{M}$ gives:

$$
V_{M}=I R_{M}
$$$$
P=I^{2} R_{M} \text {. }
$$

The power dissipation $P[$ Watts $]$ in the metal layer generates heat, causing it to reach a new equilibrium temperature $T_{L}>T_{B}$. The deposited metal layer has an electrical resistivity $\rho$, and electrical conductivity $\sigma=1 / \rho$. Each length $L$ of the wire, with cross-sectional area $A$ has a resistance $R$ set by:

$$
R=\rho L / A=(1 / \sigma)(L / A) \text {. }
$$

Two $L_{n}$ length long wires each with resistance $R_{n}$, plus one $L_{a}$ length with resistance $R_{a}$, as in (1b) of Figure 1, gives a net resistance $R_{M}$ :

$$
R_{M}=2 R_{n}+R_{a}
$$

The Wiedemann-Franz Law of Eq. [2.1.2] is used next. Let $L_{n}$ be at $T_{n}$ Kelvin, with the $L_{a}$ length at $T_{a}$, then Eq. [2.1.2] and Eq. [2.2.2] give:

$$
\begin{gathered}
R_{a}=\rho_{a} L_{a} / A_{a}=\frac{L_{a}}{\sigma_{a} w_{a} d_{o}}= \\
{\left[\left(L_{a} \Lambda_{o}\right) /\left(d_{o} K_{M}\right)\right]\left(T_{a} / w_{a}\right),} \\
R_{n}=\rho_{n} L_{n} / A_{o}=\frac{L_{n}}{\sigma_{n} w_{o} d_{o}}= \\
{\left[\left(L_{n} \Lambda_{o}\right) /\left(d_{o} K_{M}\right)\right]\left(T_{n} / w_{o}\right),} \\
\gamma_{o} \equiv\left[L_{a} / L_{n}\right], L_{a}=\gamma_{o} L_{n},
\end{gathered}
$$

where $\rho_{a} \neq \rho_{n}$ if $T_{a} \neq T_{n}$. Applying a constant voltage $V_{M}$ across the whole wire length gives a total current $I_{a}$, and a $P_{M}$ power dissipation of:

$$
\begin{aligned}
& P_{M} \equiv 2 P_{n}+P_{a}=I_{a} V_{M}= \\
& \quad\left(I_{a}\right)^{2} R_{M}=2\left(I_{a}\right)^{2} R_{n}+\left(I_{a}\right)^{2} R_{a} .
\end{aligned}
$$

The power dissipated per unit area for each metal layer section is:

$$
\begin{aligned}
P_{n} / & \left(L_{n} w_{o}\right)=\frac{K_{S}}{Z_{B}}\left(T_{n}-T_{B}\right) \\
& =\left(I_{a}\right)^{2} R_{n} /\left(L_{n} w_{o}\right) \\
& =\left(I_{a} / w_{o}\right)^{2}\left[\Lambda_{o} /\left(d_{o} K_{M}\right)\right]\left(T_{n}\right), \\
P_{a} / & \left(L_{a} w_{a}\right)=\frac{K_{S}}{Z_{B}}\left(T_{a}-T_{B}\right) \\
& =\left(I_{a}\right)^{2} R_{a} /\left(L_{a} w_{a}\right) \\
& =\left(I_{a} / w_{a}\right)^{2}\left[\Lambda_{o} /\left(d_{o} K_{M}\right)\right]\left(T_{a}\right) .
\end{aligned}
$$

These 5 equations result:

$V_{M}=\left\{2 R_{n}+R_{a}\right\} I_{a}$,

$R_{n}=\left[\frac{\Lambda_{o} L_{n}}{d_{o} K_{M}}\right]\left(\frac{T_{n}}{w_{o}}\right)_{(1)}$,

$R_{a}=\left[\frac{\Lambda_{o} L_{a}}{d_{o} K_{M}}\right]\left(\frac{T_{a}}{w_{a}}\right)_{(2)}=\left[\frac{\Lambda_{o} L_{n}}{d_{o} K_{M}}\right]\left(\frac{\gamma_{o} T_{a}}{w_{a}}\right)_{(2)}$,

$\left[\left(I_{a}\right)^{2} R_{n}\right]_{(1)}=\frac{K_{S}}{Z_{B}}\left(L_{n} w_{o}\right)\left(T_{n}-T_{B}\right)_{(1)}$,

$\left[\left(I_{a}\right)^{2} R_{a}\right]_{(2)}=\gamma_{o} \frac{K_{S}}{Z_{B}}\left(L_{n} w_{a}\right)\left(T_{a}-T_{B}\right)_{(2)},[2.2 .7 \mathrm{e}]$

with 5 unknowns $\left\{R_{a} ; R_{n} ; T_{a} ; T_{n} ; I_{a}\right\}$. The subscript [_] (1) indicates the $T_{n}$-equation, while [_] $]_{(2)}$ indicates the $T_{a}$-equation. First replacing $\left\{R_{a} ; R_{n}\right\}$ :

$V_{M}=I_{a}\left[\frac{\Lambda_{o} L_{n}}{d_{o} K_{M}}\right]\left[2\left(T_{n} / w_{o}\right)+\left(\gamma_{o} T_{a} / w_{a}\right)\right],[2.2 .8 \mathrm{a}]$

$$
\begin{gathered}
\left(I_{a}\right)^{2}\left(T_{n} / w_{o}\right)\left[\frac{\Lambda_{o} L_{n}}{d_{o} K_{M}}\right]_{(1)}= \\
\frac{K_{S}}{Z_{B}}\left(L_{n} w_{o}\right)\left[T_{n}-T_{B}\right]_{(1)}, \\
\left(I_{a}\right)^{2}\left(\gamma_{o} T_{a} / w_{a}\right)\left[\frac{\Lambda_{o} L_{n}}{d_{o} K_{M}}\right]_{(2)}= \\
\gamma_{o} \frac{K_{S}}{Z_{B}}\left(L_{n} w_{a}\right)\left[T_{a}-T_{B}\right]_{(2)}, \\
\left(I_{a}\right)^{2}\left(T_{n}\right)\left[\frac{\Lambda_{o} L_{n}}{d_{o} K_{M}}\right]_{(1)}=
\end{gathered}
$$




$$
\begin{gathered}
\frac{K_{S}}{Z_{B}}\left(L_{n} w_{o}^{2}\right)\left[T_{n}-T_{B}\right]_{(1)}, \\
\left(I_{a}\right)^{2}\left(T_{a}\right)\left[\frac{\Lambda_{o} L_{n}}{d_{o} K_{M}}\right]_{(2)}= \\
\frac{K_{S}}{Z_{B}}\left(L_{n} w_{a}^{2}\right)\left[T_{a}-T_{B}\right]_{(2)},
\end{gathered}
$$

When $w_{a} \rightarrow w_{o}$, it is the normal wire configuration of (1a) in Figure 1. Using $T_{a} \rightarrow T_{L}, T_{n} \rightarrow T_{L}$, and $I_{a} \rightarrow I_{o}$ in Eq. [2.2.8d] then gives:

$V_{M}=I_{o}\left[\frac{\Lambda_{o} L_{n}}{d_{o} K_{M}}\right]\left[2+\gamma_{o}\right]\left(T_{L} / w_{o}\right)$,

$\left(I_{o}\right)^{2}\left(T_{L}\right)\left[\frac{\Lambda_{o} L_{n}}{d_{o} K_{M}}\right]=\frac{K_{S}}{Z_{B}}\left(L_{n} w_{o}^{2}\right)\left(T_{L}-T_{B}\right) \cdot[2.2 .9 \mathrm{~b}]$

Replacing $I_{O}$ in Eq. [2.2.9b] with $V_{M}$ from Eq. [2.2.9a] gives:

$$
\begin{gathered}
\left(V_{M}\right)^{2}\left(T_{L}\right)\left[\frac{\Lambda_{o} L_{n}}{d_{o} K_{M}}\right]= \\
\left(\frac{K_{S}}{Z_{B}}\right)\left(L_{n} w_{o}^{2}\right)\left(T_{L}-T_{B}\right) \times \\
{\left[\frac{\Lambda_{o} L_{n}}{d_{o} K_{M}}\right]^{2}\left[2+\gamma_{o}\right]^{2}\left(T_{L} / w_{o}\right)^{2},} \\
\left(V_{M}\right)^{2}= \\
\Lambda_{o}\left(K_{S} / K_{M}\right)\left[\left(L_{n}\right)^{2} /\left(d_{o} Z_{B}\right)\right] \times \\
{\left[2+\gamma_{o}\right]^{2}\left(T_{L}\right)\left(T_{L}-T_{B}\right) .}
\end{gathered}
$$

For this (1a) wire case, the single parameter $\Gamma_{o}$ in Eq. [2.2.11a] sets $T_{L}$ and $\mathcal{F}_{M}$ :

$$
\begin{aligned}
& \Gamma_{o} \equiv\left(V_{M}\right)^{2} /\left\{\Lambda_{o}\left(\frac{K_{S}}{K_{M}}\right)\left[\frac{\left(L_{n}\right)^{2}}{d_{o} Z_{B}}\right]\right\}, \\
& \Gamma_{o}=\left[2+\gamma_{o}\right]^{2}\left(T_{L}\right)\left(T_{L}-T_{B}\right), \\
& \mathcal{F}_{M}\left(T_{L}\right)=\mathcal{F}_{\infty} \exp \left[-U_{M} / k_{B} T_{L}\right] .
\end{aligned}
$$

\subsection{Deviations from Nominal}

Assuming the same $V_{M}$ constant voltage conditions for both the (1a) and (1b) wire configurations in Figure 1, the $w_{a}$ anomalous width section is allowed to modify the equilibrium temperature for both the normal wire section $\left[T_{n} \neq T_{L}\right]$ and the anomalous wire section $\left[T_{a} \neq T_{L}\right]$. Using $\Gamma_{o}$ from Eq. [2.2.11a], Eqs. [2.2.8a]-[2.2.8c] become:

$$
\begin{array}{ll}
V_{M}=I_{a}\left[\frac{\Lambda_{o} L_{n}}{d_{o} K_{M}}\right]\left[\left(2 T_{n} / w_{o}\right)+\left(\gamma_{o} T_{a} / w_{a}\right)\right], & {[2.3 .1 \mathrm{a}]} \\
{\left[T_{n} \Gamma_{o}\right]_{(1)}=} & {[2.3 .1 \mathrm{~b}]} \\
{\left[\left(2 T_{n}\right)+\gamma_{o} T_{a}\left(w_{o} / w_{a}\right)\right]^{2}\left[T_{n}-T_{B}\right]_{(1)},} & {[2.3 .1 \mathrm{c}]} \\
{\left[T_{a} \Gamma_{o}\right]_{(2)}=} & {\left[2 T_{n}\left(w_{a} / w_{o}\right)+\left(\gamma_{o} T_{a}\right)\right]^{2}\left[T_{a}-T_{B}\right]_{(2)} .}
\end{array}
$$

Let $X$ be small deviation of $w_{a}$ from the normal $w_{o}$ width, which can induce a temperature change to $T_{a}$ for the $w_{a}$ geometry, as compared to $T_{n}$ for $w_{o}$ :

$$
\begin{aligned}
& w_{a} \equiv w_{o} /(1+X), \\
& T_{a} \equiv T_{n}(1+\alpha X), \\
& \text { then Eqs. }[2.3 .1 \mathrm{~b}]-[2.3 .1 \mathrm{c}] \text { become: } \\
& {\left[\Gamma_{o}\right]_{(1)}=} \\
& \quad\left[2+\gamma_{o}(1+\alpha X)(1+X)\right]^{2} T_{n}\left[T_{n}-T_{B}\right]_{(1)},
\end{aligned}
$$

$$
\left[\Gamma_{o}(1+\alpha X)\right]_{(2)}=
$$

$$
\left[\left(\frac{2}{1+X}\right)+\gamma_{o}(1+\alpha X)\right]^{2} T_{n}\left[T_{n}(1+\alpha X)-T_{B}\right]_{(2)} .
$$

When $X \rightarrow 0$, then $T_{n} \rightarrow T_{L}$, and both Eqs. [2.3.3a]-[2.3.3b] give Eq. [2.2.11b]. Normally $X$ is given, and $\left\{\alpha ; T_{n}\right\}$ are solved for. Let:

$\Delta \equiv\left(T_{n}-T_{L}\right)$

which converts Eqs. [2.3.3a]-[2.3.3b] into:

$\left[\Gamma_{o}\right]_{(1)}=\left[2+\gamma_{o}(1+\alpha X)(1+X)\right]^{2} \times$

$$
\left(T_{L}+\Delta-T_{B}\right)\left(T_{L}+\Delta\right)_{(1)},
$$

$\left[\Gamma_{o}\right]_{(2)}=\left[\left(\frac{2}{1+X}\right)+\gamma_{o}(1+\alpha X)\right]^{2} \times$

$$
\left[\left(T_{L}+\Delta\right)-\left(\frac{T_{B}}{1+\alpha X}\right)\right]\left(T_{L}+\Delta\right) .
$$

Since Eqs. [2.3.5a]-[2.3.5b] only has $(\alpha X)$ terms, $\alpha$ may not be small, but both $\{X ; \Delta\}$ can be small, so that only linear terms in either variable are needed:

$$
\begin{array}{lr}
\left(T_{L}+\Delta\right)\left(T_{L}-T_{B}+\Delta\right) \approx & {[2.3 .6 \mathrm{a}]} \\
& \left\{\left(T_{L}\right)\left(T_{L}-T_{B}\right)+\Delta\left(2 T_{L}-T_{B}\right)\right\}, \\
\left(T_{L}+\Delta\right)\left[\left(T_{L}+\Delta\right)-T_{B} /(1+\alpha X)\right] \approx & {[2.3 .6 \mathrm{~b}]} \\
& \left\{\left(T_{L}\right)\left(T_{L}-T_{B}\right)+\left[\Delta\left(2 T_{L}-T_{B}\right)+\alpha X T_{B} T_{L}\right\} .\right.
\end{array}
$$

Similarly:

$$
\begin{gathered}
\left\{\left[2+\gamma_{o}(1+\alpha X)(1+X)\right]^{2}\right\} \approx \\
\left\{\left[2+\gamma_{o}\right]^{2}+\left[2\left(2+\gamma_{o}\right)(1+\alpha) \gamma_{o} X\right]\right\}, \\
\left\{\left[2 /(1+X)+\gamma_{o}(1+\alpha X)\right]^{2}\right\} \approx \\
\quad\left\{\left[2+\gamma_{o}\right]^{2}-\left[2\left(2+\gamma_{o}\right)\left(2-\gamma_{o} \alpha\right) X\right]\right\},
\end{gathered}
$$

which converts Eqs. [2.3.5a]-[2.3.5b] into:

$\left[\Gamma_{o}\right]_{(1)} \approx$

$$
\begin{gathered}
\left\{\left[2+\gamma_{o}\right]^{2}+\left[2\left(2+\gamma_{o}\right)(1+\alpha) \gamma_{o} X\right]\right\}_{[1]} \times \\
\left\{\left(T_{L}\right)\left(T_{L}-T_{B}\right)+\Delta\left(2 T_{L}-T_{B}\right)\right\} \\
\approx\left[2+\gamma_{o}\right]^{2}\left(T_{L}\right)\left(T_{L}-T_{B}\right)+ \\
\left(T_{L}\right)\left(T_{L}-T_{B}\right)\left[2\left(2+\gamma_{o}\right)(1+\alpha) \gamma_{o} X\right]+ \\
{\left[2+\gamma_{o}\right]^{2} \Delta\left(2 T_{L}-T_{B}\right),}
\end{gathered}
$$

$\left[\Gamma_{o}\right]_{(2)} \approx$

$$
\left\{\left[2+\gamma_{o}\right]^{2}-\left[2\left(2+\gamma_{o}\right)\left(2-\gamma_{o} \alpha\right) X\right]\right\}_{[2]} \times
$$

$\left\{\left(T_{L}\right)\left(T_{L}-T_{B}\right)+\left[\Delta\left(2 T_{L}-T_{B}\right)+\alpha X T_{B} T_{L}\right]\right\}_{[2]}$ $\approx\left[2+\gamma_{o}\right]^{2}\left(T_{L}\right)\left(T_{L}-T_{B}\right)+$

$$
\left[2+\gamma_{o}\right]^{2}\left[\Delta\left(2 T_{L}-T_{B}\right)+\alpha X T_{B} T_{L}\right]
$$$$
-\left(T_{L}\right)\left(T_{L}-T_{B}\right)\left[2\left(2+\gamma_{o}\right)\left(2-\gamma_{o} \alpha\right) X\right],
$$

Using Eq. $[2.2 .11 \mathrm{~b}]$ to subtract out $\Gamma_{o}$ gives:

$$
\begin{gathered}
0 \approx\left(2+\gamma_{o}\right)\left[\Delta\left(2 T_{L}-T_{B}\right)\right]_{(1)} \quad[2.3 .9 \mathrm{a}] \\
\quad+X\left(T_{L}\right)\left(T_{L}-T_{B}\right)\left[2(1+\alpha) \gamma_{o}\right]_{(1)}, \\
0 \approx\left(2+\gamma_{o}\right)\left[\Delta\left(2 T_{L}-T_{B}\right)+\alpha X T_{B} T_{L}\right]_{(2)}[2.3 .9 \mathrm{~b}]
\end{gathered}
$$

$$
-X\left(T_{L}\right)\left(T_{L}-T_{B}\right)\left[2\left(2-\gamma_{o} \alpha\right)\right]_{(2)} \text {. }
$$

As the $\Delta$-terms match, matching $X$-terms sets $\alpha$ :

$$
\left(T_{L}\right)\left(T_{L}-T_{B}\right)\left[2(1+\alpha) \gamma_{o}\right]_{[1]}=
$$

$\left\{\left(2+\gamma_{o}\right)\left(\alpha T_{B} T_{L}\right)-\left(T_{L}\right)\left(T_{L}-T_{B}\right)\left[2\left(2-\gamma_{o} \alpha\right)\right]\right\}_{[2]}$,

$\left(2+\gamma_{o}\right)\left(\alpha T_{B} T_{L}\right)=$

[2.3.10b] 


$$
\begin{aligned}
& 2\left(T_{L}\right)\left(T_{L}-T_{B}\right)\left[(1+\alpha) \gamma_{o}+\left(2-\gamma_{o} \alpha\right)\right] \\
& =2\left(T_{L}\right)\left(T_{L}-T_{B}\right)\left[2+\gamma_{o}\right], \\
& (\alpha)=2\left(T_{L}-T_{B}\right) / T_{B} . \\
& =2 \gamma_{o}\left(T_{L} / T_{B}\right)\left(T_{L}-T_{B}\right)\left(2 T_{L}-T_{B}\right) \text {, } \\
& T_{a} \equiv T_{n}(1+\alpha X)=T_{n}\left[1+\frac{2\left(T_{L}-T_{B}\right)}{T_{B}} X\right][2.3 .13 \mathrm{a}] \\
& =T_{L}\left[1-2\left(\frac{\gamma_{o}}{2+\gamma_{o}}\right)\left(\frac{T_{L}-T_{B}}{T_{B}}\right) X\right]\left[1+\frac{2\left(T_{L}-T_{B}\right)}{T_{B}} X\right] \\
& \approx T_{L}\left\{1+\left[\frac{2\left(T_{L}-T_{B}\right)}{T_{B}}\right]\left[\frac{2}{2+\gamma_{o}}\right] X\right\}, \\
& \frac{\left(T_{a}-T_{L}\right)}{T_{L}}=+\left(\frac{2}{2+\gamma_{o}}\right)\left[\frac{2\left(T_{L}-T_{B}\right)}{T_{B}}\right] X \text {. }
\end{aligned}
$$

Combining Eq. [2.3.12b] and Eq. [2.3.13b] gives:

$$
\begin{aligned}
& \frac{\left(T_{a}-T_{n}\right)}{T_{L}}= \\
& \quad\left[\frac{2\left(T_{L}-T_{B}\right)}{T_{B}}\right]\left[\frac{2}{2+\gamma_{o}}\right] X+\left(\frac{\gamma_{o}}{2+\gamma_{o}}\right)\left[\frac{2\left(T_{L}-T_{B}\right)}{T_{B}}\right] X,
\end{aligned}
$$$$
\left(T_{a}-T_{n}\right)=2\left(\frac{T_{L}}{T_{B}}\right)\left(T_{L}-T_{B}\right) X .
$$

When $X \rightarrow 0$, then $w_{a} \rightarrow w_{o}$, and $T_{a} \rightarrow T_{n} \rightarrow T_{L}$. Since the temperatures $\left\{T_{L} ; T_{B}\right\}$ for the normal (1a) wire and heat-sink are assumed known, Eq. [2.3.14b] shows the temperature difference between the two sections of the (1b) anomalous wire configuration is a direct measure of the fractional deviation from normal for the anomalous wire geometry.

\subsection{Lognormal Completion}

Given a wire of length $\left(2 L_{n}+L_{a}\right)$ atop a substrate, as in Figure 1, with a heat-sink at depth $Z_{B}$ and temperature $T_{B}$, applying a $V_{M}$ operating voltage across the normal wire configuration (1a) gives a nominal $T_{L}$ wire temperature and failure rate $\mathcal{F}_{M}$ :

$\mathcal{F}_{M}\left(T_{L}\right)=\mathcal{F}_{\infty} \exp \left[-U_{M} / k_{B} T_{L}\right]$.

Let the anomalous wire (1b) have a section anomalous $w_{a}$ width of:

$$
\begin{aligned}
& w_{a} \equiv w_{o} /(1+X), \\
& \gamma_{o} \equiv\left[L_{a} / L_{n}\right],
\end{aligned}
$$

extending over a length $L_{a}$. Assuming that $X$ is small, corrections of $\vartheta\left(X^{2}\right)$ can be ignored. For $X>0$, the $w_{a}$ section will reach its own new equilibrium temperature $T_{a}>T_{L}$, giving a new failure rate $\mathcal{F}_{M}\left(T_{a}\right)$ :

$$
\begin{array}{cl}
T_{a}=T_{L}\left\{1+\left(\frac{2}{2+\gamma_{o}}\right)\left[\frac{2\left(T_{L}-T_{B}\right)}{T_{B}}\right] X\right\}, & \\
\mathcal{F}\left(T_{a}\right)=\mathcal{F}_{M} \exp \left[-U_{M}\left(\frac{1}{k_{B} T_{a}}-\frac{1}{k_{B} T_{L}}\right)\right] . & \\
\ln \left[\mathcal{F}\left(T_{a}\right) / \mathcal{F}_{M}\right]= & \\
\quad-U M\left(\frac{1}{k_{B} T_{a}}-\frac{1}{k_{B} T_{L}}\right)=+\frac{U M}{k_{B} T_{a}}\left(\frac{T_{a}-T_{L}}{T_{L}}\right) \\
\quad=+\frac{U M}{k_{B} T_{a}}\left[\frac{2\left(T_{L}-T_{B}\right)}{T_{B}}\right]\left[\frac{2}{2+\gamma_{o}}\right] X-\vartheta\left(X^{2}\right) .
\end{array}
$$

In contrast, the two normal geometry wire lengths in the anomalous wire (1) $)$, each of length $L_{n}$ and width $w_{o}$, will have a cooler temperature $T_{n}<T_{L}$ :

$$
\begin{array}{ll}
\frac{\left(T_{n}-T_{L}\right)}{T_{L}}=-\left[\frac{2\left(T_{L}-T_{B}\right)}{T_{B}}\right]\left[\frac{\gamma_{o}}{2+\gamma_{o}}\right] X, & {[2 .} \\
T_{n}=T_{L}\left\{1-\left[\frac{2\left(T_{L}-T_{B}\right)}{T_{B}}\right]\left[\frac{\gamma_{o}}{2+\gamma_{o}}\right] X\right\}, & {[2.4} \\
\mathcal{F}\left(T_{n}\right)=\mathcal{F}_{M} \exp \left[-U M\left(\frac{1}{k_{B} T_{n}}-\frac{1}{k_{B} T_{L}}\right)\right] . & {[2.4} \\
\ln \left[\mathcal{F}\left(T_{n}\right) / \mathcal{F}_{M}\right]= & {[2.4} \\
\quad-U M\left(\frac{1}{k_{B} T_{n}}-\frac{1}{k_{B} T_{L}}\right)=+\frac{U M}{k_{B} T_{n}}\left(\frac{T_{n}-T_{L}}{T_{L}}\right) \\
\quad=-\frac{U M}{k_{B} T_{L}}\left[\frac{2\left(T_{L}-T_{B}\right)}{T_{B}}\right]\left[\frac{\gamma_{o}}{2+\gamma_{o}}\right] X-\vartheta\left(X^{2}\right),
\end{array}
$$

showing both Eq. [2.4.3c] and Eq. [2.4.4c] depend on the $\left\{L_{a} ; L_{n}\right\}$ lengths due to their $\gamma_{o}$-term. However, the companion to Eq. [2.3.14b]:

$$
\begin{aligned}
& \ln \left[\mathcal{F}\left(T_{a}\right) / \mathcal{F}\left(T_{n}\right)\right]= \\
& \quad+\frac{U M}{k_{B} T_{L}}\left[\frac{2\left(T_{L}-T_{B}\right)}{T_{B}}\right] X-\vartheta\left(X^{2}\right),
\end{aligned}
$$

is independent of those lengths, and is primarily a function of the geometry changes, as set by $X$ in Eq. [2.4.2a]. When those geometry changes are due to random manufacturing deviations about a mean, $X$ is then a random variable, with zero mean and a given standard deviation. When $X$ is a Gaussian, then the failure rates $\left\{\mathcal{F}\left(T_{a}\right) ; \mathcal{F}\left(T_{n}\right)\right\}$ and the Eq. [2.4.5] ratio, all become Lognormal distributions.

\section{Summary}

When a wire connection is established by depositing a metal layer atop a substrate, the dominant wire failure mechanism is likely due to an unwanted gradual interaction between the wire and its substrate, which is likely diffusion dominated. The wire failure rate $\mathcal{F}$ 
then depends on its nominal operating temperature $T_{L}$, and an activation energy $U_{M}$ :

$$
\mathcal{F}_{M}\left(T_{L}\right)=\mathcal{F}_{\infty} \exp \left[-U_{M} / k_{B} T_{L}\right] .
$$

The normal wire configuration top view is shown as (1a) in Figure 1, which has a uniform metal layer width $w_{o}$. A side view is shown in Figure 2, with the metal layer of thickness $d_{o}$ at $Z=0$ atop a substrate of thickness $Z_{B}$, while a heat-sink at $Z=Z_{B}$ maintains a constant temperature $T_{B}$ plane.

The Wiedemann-Franz Law ${ }^{7}$ sets $\mathcal{F}_{M}$ and $T_{L}$ for the normal wire configuration of (1a) in Figure 1, along with this Eq. [3.0.2a] $\Gamma_{o}$ lumped parameter:

$$
\begin{array}{ccc}
\Gamma_{o} \equiv & {[3.0 .2 \mathrm{a}]} \\
& \left(V_{M}\right)^{2} /\left\{\Lambda_{o}\left(K_{S} / K_{M}\right)\left[\left(L_{n}\right)^{2} /\left(d_{o} Z_{B}\right)\right]\right\}, \\
\Gamma_{o}= & {\left[2+\gamma_{o}\right]^{2}\left(T_{L}\right)\left(T_{L}-T_{B}\right),} & {[3.0 .2 \mathrm{~b}]}
\end{array}
$$

where $K_{M}$ and $K_{S}$, respectively, are the metal layer and substrate thermal conductivities $[$ Watts / $($ meter $-K)]$, and $\Lambda_{o}$ is the Lorentz Number:

$$
\Lambda_{o}=\frac{\pi^{2}}{3}\left(\frac{k_{B}}{e}\right)^{2} \approx 2.445 \times 10^{-8} \frac{\text { Watt-ohms }}{(K)^{2}} \text {. [3.0.3] }
$$

Under constant $V_{M}$ voltage conditions, let a wire section of length $L_{a}$ have a different deposited metal layer width $w_{a}[(\mathbf{1 b})$ in Figure 1$]$, abutted by two normal geometry regions of $\left\{L_{n} ; w_{o}\right\}$ length and width. Both the anomalous $\left\{L_{a} ; w_{a}\right\}$ region and the adjacent normal $\left\{L_{n} ; w_{o}\right\}$ regions will reach new equilibrium temperatures $\left\{T_{a} ; T_{o}\right\}$ respectively, compared to (1a) in Figure 1. The following modified local failure rates then result:

$$
\begin{array}{ll}
\mathcal{F}\left(T_{a}\right)=\mathcal{F}_{M} \exp \left[-U_{M}\left(\frac{1}{k_{B} T_{a}}-\frac{1}{k_{B} T_{L}}\right)\right], & {[3.0 .4 \mathrm{a}]} \\
\mathcal{F}\left(T_{n}\right)=\mathcal{F}_{M} \exp \left[-U_{M}\left(\frac{1}{k_{B} T_{n}}-\frac{1}{k_{B} T_{L}}\right)\right] . & {[3.0 .4 \mathrm{a}]} \\
\text { Using } X \text { as a measure of the } w_{a} \text { deviation from its }
\end{array}
$$
normal $w_{o}$ value:

$$
\begin{aligned}
& w_{a} \equiv w_{o} /(1+X) \text {, } \\
& \gamma_{o} \equiv\left[L_{a} / L_{n}\right] \\
& \text { for small } X \text {, ignoring terms of } \vartheta\left(X^{2}\right) \text {, we showed: } \\
& \left(T_{a}-T_{n}\right)=2\left(\frac{T_{L}}{T_{B}}\right)\left(T_{L}-T_{B}\right) X, \quad \text { [3.0.6a] } \\
& \ln \left[\mathcal{F}\left(T_{a}\right) / \mathcal{F}_{M}\right]= \\
& +\frac{U_{M}}{k_{B} T_{L}}\left[\frac{2\left(T_{L}-T_{B}\right)}{T_{B}}\right]\left[\frac{2}{2+\gamma_{o}}\right] X, \\
& \ln \left[\mathcal{F}\left(T_{n}\right) / \mathcal{F}_{M}\right]= \\
& -\frac{U M}{k_{B} T_{L}}\left[\frac{2\left(T_{L}-T_{B}\right)}{T_{B}}\right]\left[\frac{\gamma_{o}}{2+\gamma_{o}}\right] X,
\end{aligned}
$$

If the Eq. [3.0.5a] geometry changes are due to random manufacturing deviations about a mean, $X$ becomes a random variable with a standard deviation and zero mean. When $X$ is a Gaussian, then the failure rates $\mathcal{F}\left(T_{a}\right)$ and $\mathcal{F}\left(T_{n}\right)$, and the Eq. [3.0.6d] ratio, all become Lognormal distributions.

\section{References}

1. A. S. Jordan, R. H. Peaker, R. H. Saul, H. J. Braun, and H. H. Wade, "The Reliability of 302A Numerics", The Bell System Technical Journal, Vol. 57, No. 8, October 1978.

2. doi.org/10.1016/0026-2714(78)90331-1, A. S. Jordan, "A Comprehensive Review of the Lognormal Failure Distribution with Application to LED Reliability", Microelectronics Reliability 18(3), 1978, pp. 267-279.

3. D. S. Peck and C. H. Ziedt Jr., "The Reliability of Semiconductor Devices in the Bell System," Proc. IEEE 62, 185-211 (1974).

4. F. H. Reynolds, "Thermally Accelerated Aging of Semiconductor Components," Proc. IEEE 62, 212-222 (1974).

5. W. A. Johnson and M. F. Millea, "Statistical Analysis of Accelerated Temperature Aging of Semiconductor Devices", The Aerospace Corporation, Report No. SD-TR-81-37, 15 May 1981, USG Accession No. AD-A099439.

6. J. Aitchison and J. A. C. Brown, The Lognormal Distribution with special reference to its uses in economics, 176 pages. University of Cambridge Department of Applied Economics, Monographs Volume 5, Cambridge University Press (1957).

7. tnasc.com/wp-content/ uploads/2021/09/Jour4-11-26-R.pdf

V. Devanathan, "The Wiedemann-Franz Law for Electrical and Thermal Conduction in Metals", J. Chennai Acad. Sci. 4, pp, 1-26 (2021). 